\title{
Deficiency in Heat Shock Factor 1 (HSF-1) Expression Exacerbates Sepsis-induced Inflammation and Cardiac Dysfunction
}

\author{
Robert C Barber' ${ }^{1}$, David L Maass ${ }^{2}$, D Jean White ${ }^{2}$, Jureta W Horton ${ }^{2}$, Steven E Wolf ${ }^{2}$, Joseph P \\ Minei $^{2}$ and Qun S Zang ${ }^{2 *}$ \\ ${ }^{1}$ University of North Texas Health Science Center, Department of Pharmacology and Neurosciences, Fort Worth, TX, USA \\ ${ }^{2}$ Department of Surgery, University of Texas Southwestern Medical Center, 5323 Harry Hines Blvd, Dallas, TX 75390, USA
}

Received: November 25, 2013; Accepted: January 10, 2014; Published: January 27, 2014

*Corresponding author: Qun S Zang, Department of Surgery, UT Southwestern Medical Center, 5323 Harry Hines Blvd, Dallas, TX 75390-9160, USA, Tel: 214-648-5442; Fax: 214-648-8420; Email: qun.zang@utsouthwestern.edu

\begin{abstract}
In the present study, we investigated whether absence of heat shock factor 1 (HSF-1) and inability to increase myocardial expression of heat shock proteins alter septic responses of inflammatory cytokines and myocardial contractility. HSF-1 knockout $\left(h s f^{\prime}\right)$ mice and wild type litter mates underwent a sterile (lipopolysaccharide; LPS) or infectious (Streptococcus pneumoniae or Klebsiella pneumoniae) septic challenge. Production of cytokines, TNF, IL-1 $\beta$, IL-6 and IL-10, in the blood and from cardiomyocytes was exaggerated in the $h s f^{\prime}$ mice compared to responses measured in wild type mice given an identical septic challenge. This enhanced compartmentalized myocardial inflammation was associated with significantly decreased cardiac contraction and diminished relaxation in the $h s f^{\prime-}$ mice. However, lacking HSF-1 expression did not affect intracellular calcium and sodium responses in cardiomyocytes isolated from septic challenged mice, suggesting that ion loading was not a major or sustaining cause of the greater myocardial contractile defects in $h s f^{/}$mice. In conclusion, our data indicated that HSF-1 and downstream heat shock proteins are essential components to support cardiac function in sepsis. Further studies are warranted to further define the precise mechanisms of HSF-1 mediated cardiac protection.
\end{abstract}

Keywords: HSF-1; Heat shock proteins; Sepsis; Cardiac dysfunction; Inflammation; Cytokines; Cesium handling

\section{Introduction}

Severe sepsis is a leading cause of death in intensive care units $[1,2]$. Despite improvements in antibiotic therapies and critical care techniques [3], approximately 215,000 Americans still die from sepsis each year [4]. Present treatment for sepsis continues to be supportive care and source control, such as using intravenous fluids and oxygen, and/or antibiotics and procedural interventions [3]. Most attempts at molecule-based treatments have failed clinically $[5,6]$. To date, our understanding of sepsis pathogenesis and therapeutic options are still limited. Therefore, investigation of the pathological mechanisms and exploration of new therapeutic interventions are clearly needed to advance the treatment of this devastating condition.
Cardiac dysfunction is a vital component of multi-organ failure during severe sepsis [7-9]. Increased cardiac expression of heat shock protein 70 (HSP70) has been described after ischemic injury [10], hypoxia [11] and major burn [12]. A cardioprotective role has been suggested for HSP70, which is believed to function as a molecular chaperone that inhibits apoptosis and necrosis secondary to inhibitory effects on caspases [13]. HSPs may also provide cardioprotection through their ability to interact with cytoskeletal proteins by stabilizing cytoskeletal structures and increasing resistance to stress $[14,15]$. HSP70 expression after injury or stress is regulated by heat shock transcription factor-1 (HSF-1) [16]. Stress promotes HSF1 conversion from a monomeric form in the cytoplasm to a trimerized phosphorylated form, which then translocates to the nucleus to promote the transcription of heat shock proteins [17]. In the heart, HSF1 deficiency reduces cardiac expression of Hsp25, alphaB-crystallin and Hsp70 [16]. The role of HSF-1/ HSP70 in inflammation has been emphasized by the finding that HSF-1 deficient mice exhibit chronically elevated systemic TNF levels as well as increased susceptibility to LPS challenge [18]. In this study, using HSF-1 knockout (hsf/-) mice as a model, we examined the role of HSF-1 in inflammation and cardiac function in response to septic challenge by lipopolysaccharide (LPS), grampositive bacteria Streptococcus pneumoniae (S. pneumoniae), or gram-negative bacteria Klebsiella pneumoniae (K. pneumoniae).

\section{Materials and Methods}

\section{Experimental animals and sepsis models}

HSF-1 heterozygous knockout mice were obtained from Dr. Ivor Benjamin at University of Texas Southwestern Medical Center (UTSW) $[19,20]$. Males and females of these mice were intercrossed to produce homozygous knockout, $h s f-1^{\%}\left(H s f 1^{\text {tmlljb }}\right)$, mice, and gene knockout was confirmed by RT-PCR.

Male $\mathrm{Hsf-1 \%}$ mice and wild type litter mates (C57BL/6, $\mathrm{Hsf}$ $1^{+/+}$), 8-10 weeks of age and body weight 20-22g for wild type or $15-18 \mathrm{~g}$ for $H s f-1 \%$, were subjected to three distinct septic 
challenges: (1) lipopolysaccharide (LPS), (2) gram-positive bacteria Streptococcus pneumoniae, type 3, and (3) gram-negative bacteria Klebsiella pneumoniae. Among these models, LPS and its control vehicle were given via intraperitoneal (IP) administration at the dose of $4 \mathrm{mg} / \mathrm{kg}$ (in $20 \mu \mathrm{L}$ saline as vehicle). The mice were subjected to bacterial infection via intratracheal (IT) delivery of bacterial resuspension at $1 \times 10^{5} \mathrm{CFU}$ per mouse (in $0.3 \mathrm{~mL}$ sterile PBS as vehicle). The IT inoculation procedure was performed according to our previously established protocol [2123], and blood samples were collected 24 hours after inoculation to confirm the presence of bacteremia. All mice received IP resuscitation (lactated Ringer's solution, $2 \mathrm{~mL}$ ), and were studied $24 \mathrm{hrs}$ after septic challenge.

In the present study, animal protocol and pathogen safety plans were approved by Institutional Animal Care and Use Committee (IACUC) and the Department of Environmental Health and Safety (EH\&S) at the University of Texas Southwestern Medical Center (UTSW). All work described was performed according to the current guidelines for caring animals and handling biohazard agents.

\section{Isolation and culture of primary cardiomyocytes}

Isolation of cardiomyocytes from adult mice was performed as previously described [24-26]. The mice were briefly given an intraperitoneal injection of heparin, 500 units/mouse, 20-30 minutes before they were sacrificed by decapitation. Hearts were harvested and placed in a petri dish containing heart medium at room temperature [in mM: $113 \mathrm{NaCl}, 4.7 \mathrm{KCl}, 0.6 \mathrm{KH}_{2} \mathrm{PO}_{4}, 0.6$ $\mathrm{Na}_{2} \mathrm{HPO}_{4}, 1.2 \mathrm{MgSO}_{4}, 12 \mathrm{NaHCO}_{3}, 10 \mathrm{KHCO}_{3}, 20 \mathrm{D}$-glucose, $10 \mathrm{HEPES}$ buffer solution, 30 taurine, 2 carnitine, 2 creatine, and plus $0.5 \mathrm{x}$ MEM amino acids (Invitrogen, Carlsbad, CA)], which was bubbled constantly with $95 \% \mathrm{O}_{2}-5 \% \mathrm{CO}_{2}$. Hearts were cannulated via the aorta and perfused with heart medium at a rate of $1 \mathrm{~mL} / \mathrm{min}$ for 5 minutes in a nonrecirculating mode. Enzymatic digestion was initiated by perfusing the heart with digestion solution [prepared by adding $50 \mathrm{mg}$ of collagenase II (Worthington Biochemical Corporation, Lakewood, NJ) and $50 \mathrm{mg}$ bovine serum albumin (BSA), Fraction V (Invitrogen, Carlsbad, CA) to $34.5 \mathrm{~mL}$ of heart medium, plus $1 \mathrm{x}$ trypsin (Invitrogen, Carlsbad, CA), $15 \mu \mathrm{M} \mathrm{CaCl}_{2}$, and $40 \mathrm{mM}$ 2,3-butanedione monoxime (BDM)]. Enzymatic digestion was accomplished by recirculating this solution through the heart at a flow rate of $1 \mathrm{~mL} / \mathrm{min}$ for 20 minutes. All solutions perfusing the heart were maintained at a constant $37^{\circ} \mathrm{C}$. At the end of the enzymatic digestion, the ventricles were removed and mechanically disassociated in $6 \mathrm{~mL}$ of enzymatic digestion solution containing a $6-\mathrm{mL}$ aliquot of $2 \times$ BDM/BSA solution [ $3 \mathrm{mg}$ $\mathrm{BSA}$, Fraction $\mathrm{V}$ to $150 \mathrm{~mL}$ of BDM stock, $40 \mathrm{mM}$ ]. After mechanical disassociation with fine forceps, the tissue homogenate was filtered through a mesh filter into a conical tube. Cells adherent to the filter were collected by washing with an additional $10 \mathrm{ml}$ aliquot of $1 \times$ BDM/BSA solution [prepared by combining $100 \mathrm{~mL}$ of BDM stock, $40 \mathrm{mM} ; 100 \mathrm{~mL}$ of heart medium; and $2 \mathrm{~g}$ of BSA, Fraction V]. Cells were then allowed to pellet in the conical tube for 10 minutes. The supernatant liquid was removed, and the pellet was re-suspended in $10 \mathrm{~mL}$ of $1 \times$ BDM/BSA. The cells were washed and pelleted further in BDM/BSA buffer with increasing concentrations of calcium $(100 ; 200 ; 500$; and $1,000 \mu \mathrm{M})$. After the final pelleting step, the supernatant liquid was removed, and the pellet was re-suspended in MEM [contains 1× MEM (SigmaAldrich, St. Louis, MO); 11.9 mM NaHCO $; 10$ mM HEPES; and 1X penicillin/streptomycin (Invitrogen, Carlsbad, CA)]. At the time of MEM preparation, the medium was bubbled with $95 \% \mathrm{O}_{2}$ $5 \% \mathrm{CO}_{2}$ for 15 minutes, and the $\mathrm{pH}$ was adjusted to 7.1 with $1 \mathrm{M}$ $\mathrm{NaOH}$. The solution was then sterilized by filtration and stored at $4^{\circ} \mathrm{C}$ until use. At the final concentration of calcium, the cell viability was measured, and only cell suspensions with greater than $85 \%$ viability were used for subsequent studies.

To culture, cardiomyocytes were plated at a density of $10^{6}$ cells/plate on $100 \mathrm{~mm}$ petri dishes and incubated with MEM medium at $37^{\circ} \mathrm{C}$ incubator. Cells were harvested $12-18$ hours after culture.

\section{Measurement of cytokines}

In blood serum and in the conditional medium of primary cardiomyocyte cultures, levels of TNF, IL-1 $\beta$, IL- 6 and IL-10 were measured using immunoassay kits (Biosource, Camarillo, CA). Blood was collected from sacrificed animals using Vacutainer rapid serum tubes (RST) (BD Diagnostics, Franklin Lakes, NJ) followed by immediate centrifugation at 3,000g for $15 \mathrm{~min}$ at $4{ }^{\circ} \mathrm{C}$, for preparing serum. The serum preparations were then allocated and stored at $-80^{\circ} \mathrm{C}$ until analyzed. Cardiomyocyte medium was collected when isolated myocytes were cultured at $1 \times 10^{5} / \mathrm{mL}$ per well on 12 -well plates (Corning, Corning, NY) for 18 hours.

\section{Measurement of intracellular calcium and sodium levels}

Levels of intracellular calcium and sodium were measured in primary cardiomyocytes harvested from all experimental groups. Cells were loaded with either fluorescent calcium probe Fura-2 AM (Sigma-Aldrich, St. Louis, MO) for 45 min or sodium-binding benzofuran isophthalate (SBFI) (Sigma-Aldrich, St. Louis, MO) for $60 \mathrm{~min}$ at room temperature in the dark. Myocytes were then suspended in $1.88 \mathrm{mM}$ calcium-containing Tyrodes solution and washed to remove extracellular dye; myocytes were placed in a perfusion chamber on the stage of a Nikon inverted microscope which was interfaced with Grooney ${ }^{\mathrm{TM}}$ optics for epi-illumination, a triocular head, phase optics, and a 10X phase-contrast objective and mechanical stage. The excitation illumination source (300 W compact Xenon arc illuminator) was equipped with a power supply. In addition, this InCyt $\operatorname{Im} 2^{\mathrm{TM}}$ Fluorescence Imaging System (Intracellular Imaging, Cincinnati, OH) included an imaging workstation and computer. The computer-controlled filter changer allowed alternation between the 340 and $380 \mathrm{~nm}$ excitation wavelengths. Images were captured by a monochrome charge coupled device camera equipped with a TV relay lens. InCyt $\operatorname{Im} 1^{\mathrm{TM}}$ and InCyt Im2 $2^{\mathrm{TM}}$ Image software allowed measurement of the intracellular calcium and sodium concentrations from the ratio of the fluorescent signals generated at the two excitation wavelengths; auto fluorescence of myocytes that had not been loaded with fluorescent dye was measured with each experiment and subtracted. The calibration procedure included measuring 
the fluorescence ratio with buffers having different calcium or sodium concentrations. At each wavelength, the fluorescence emissions were collected for $3 \mathrm{msec}$ intervals, and the time between data collections was $12 \mathrm{msec}$. Because quiescent or noncontracting myocytes were used in these studies, the calcium and sodium concentrations measured reflect those in diastole.

\section{Analysis of cardiac function}

As previously described, cardiac contraction and relaxation were examined using Langendorf heart perfusions [27]. Mice were anticoagulated with sodium heparin (200 U; Elkins-Sinn, Inc., Cherry Hill, NJ) and killed by decapitation. The heart was removed rapidly and placed in a petri dish containing ice-cold $\left(4^{\circ} \mathrm{C}\right)$ Krebs-Henseleit bicarbonate-buffered solution (in $\mathrm{mM}$ : $\mathrm{NaCl}$ 118, $\mathrm{KCl} 4.7, \mathrm{NaHCO}_{3} 21, \mathrm{CaCl}_{2} 2.5, \mathrm{MgSO}_{4} 1.2, \mathrm{KH}_{2} \mathrm{PO}_{4} 1.2$, and glucose 11). All solutions were prepared each day with demineralized, deionized water and bubbled with $95 \% \mathrm{O}_{2}-5 \%$ $\mathrm{CO}_{2}$ ( $\mathrm{pH} 7.4 ; \mathrm{pO}_{2} 550 \mathrm{~mm} \mathrm{Hg} ; \mathrm{pCO}_{2} 38 \mathrm{~mm} \mathrm{Hg}$ ). A cannula placed in the ascending aorta was connected via glass tubing to a bufferfilled reservoir for perfusion of the coronary circulation at a constant flow rate. Hearts were suspended in a temperaturecontrolled chamber maintained at $38 \pm 0.5^{\circ} \mathrm{C}$, and a constantflow pump (Holter, Model 911; Critikon, Inc., Tampa, FL) was used to maintain perfusion of the coronary artery by retrograde perfusion of the aortic stump cannula as previously described $[28,29]$. Coronary perfusion pressure was measured, and effluent was collected to confirm the coronary flow rate. Left ventricular pressure (LVP) was measured with a Statham pressure transducer (Model P23ID; Gould Instruments Inc., Oxnard, CA) attached to the cannula placed in the left ventricle, and the rate of LVP rise $(+\mathrm{dP} / \mathrm{dt})$ and fall $(-\mathrm{dP} / \mathrm{dt})$ were obtained using an electronic differentiator (Model 7P20C; Grass Instruments, Inc., Quincy, MA) and recorded (Model 7DWL8P, Grass Instruments). The LVP and $\pm \mathrm{dP} / \mathrm{dt}$ responses to increase in perfusate calcium were also examined. Data input from the Grass recorder was transferred to a computer and a Grass Poly VIEW Data Acquisition System was used to convert data into digital form.

\section{Statistical analysis}

All data were expressed as mean \pm SEM of at least 3 independent experiments using 4-8 animals/group. Student's t-tests were used to assess the difference between the sham and the various sepsis groups. Because two outcome measures (wild type and knockout) were tested against three hypothesized predictors (sepsis by LPS, S. pneumoniae or K. pneumoniae), a Bonferroni's-adjusted probability value less than 0.0083 was considered statistically significant.

\section{Results}

In the present study, wild type and $h s f^{/-}$mice were given sham or septic challenge by LPS, gram-positive bacteria Streptococcus pneumoniae (S. pneumoniae), or gram-negative bacteria Klebsiella pneumoniae ( $K$. pneumonia). Animals in all groups survived 24 hours of septic challenge.

\section{Cytokine production}

To evaluate the effect of HSF-1 deficiency on systemic inflammation, cytokine levels were measured in the blood serum collected 24 hours after septic challenge (or sham challenge). As summarized in Figure 1, in response to sham septic challenge, absence of HSF-1 did not cause significant changes in the basal levels of TNF, IL-1 $\beta$, IL-6, and IL-10 in circulation. Both LPS and bacterial infection, either by $S$. pneumoniae or K. pneumonia, induced a significant rise of cytokines in the wild type mice. Further, $h s f^{/}$mice presented amplified cytokine responses in peripheral blood compared with their wild type counter parts, $(p<0.05)$.

We then estimated whether HSF-1 deficiency alters inflammation in myocardium. Levels of cytokines secreted from primary cardiomyocytes isolated from wild type and $h s f^{/-}$mice receiving sham or septic challenge were compared. The absence of HSF-1 did not alter the production of IL-1, IL-6, IL-10 and TNF in cardiomyocytes from sham subjects (Figure 2). However, a robust secretion of cytokines was detected in all cardiomyocytes isolated from mice given LPS challenge or bacterial infection. Similar to what we observed in circulating cytokine responses (Figure 1), LPS, S. pneumoniae or K. pneumoniae triggered significantly higher levels of cytokine productions in myocytes from $h s f^{/}$animals, compared with the cells isolated from the wild type mice.

In previous studies, we confirmed that fewer than $2 \%$ of the total cells in a cardiomyocyte preparation were noncardiomyocytes using this technique; therefore, a majority of the inflammatory cytokines measured in the cardiomyocyte medium was indeed cardiomyocyte derived [30]. The impact of HSF-1 deficiency on cytokine levels in wild type versus $h s f^{\prime-}$ mice suggests that HSF-1 plays a significant role in control of both systemic and myocardial inflammation during sepsis.

\section{Intracellular calcium and sodium levels in cardiomyocytes}

Our previous studies have suggested that, in cardiomyocytes, changes in calcium and sodium flux affect contractility as well as the inflammatory responses [31-33]. In this report, we measured calcium and sodium levels in the cardiomyocytes isolated $24 \mathrm{hrs}$ after septic challenge by LPS, S. pneumoniae or K. pneumoniae. In these cells, intracellular calcium (Figure $3 \mathrm{~A}$ ) and sodium (Figure 3B) levels were similar in all mice in the absence of septic challenge. Septic challenge by LPS, $S$. pneumoniae or $K$. pneumoniae promoted calcium and sodium loading in myocytes, however, we found no significant differences detected between wild type and $h s f^{/-}$mice. With regard to intracellular sodium (Figure 3B), the difference between LPS-challenged wild type and knockout mice was subtle and repeatable, however not statistically significant.

\section{Cardiac function analysis}

We next explored the functional consequence of HSF-1 deficiency in sepsis-induced cardiac dysfunction. According to previously established protocol, we applied isolated hearts from 

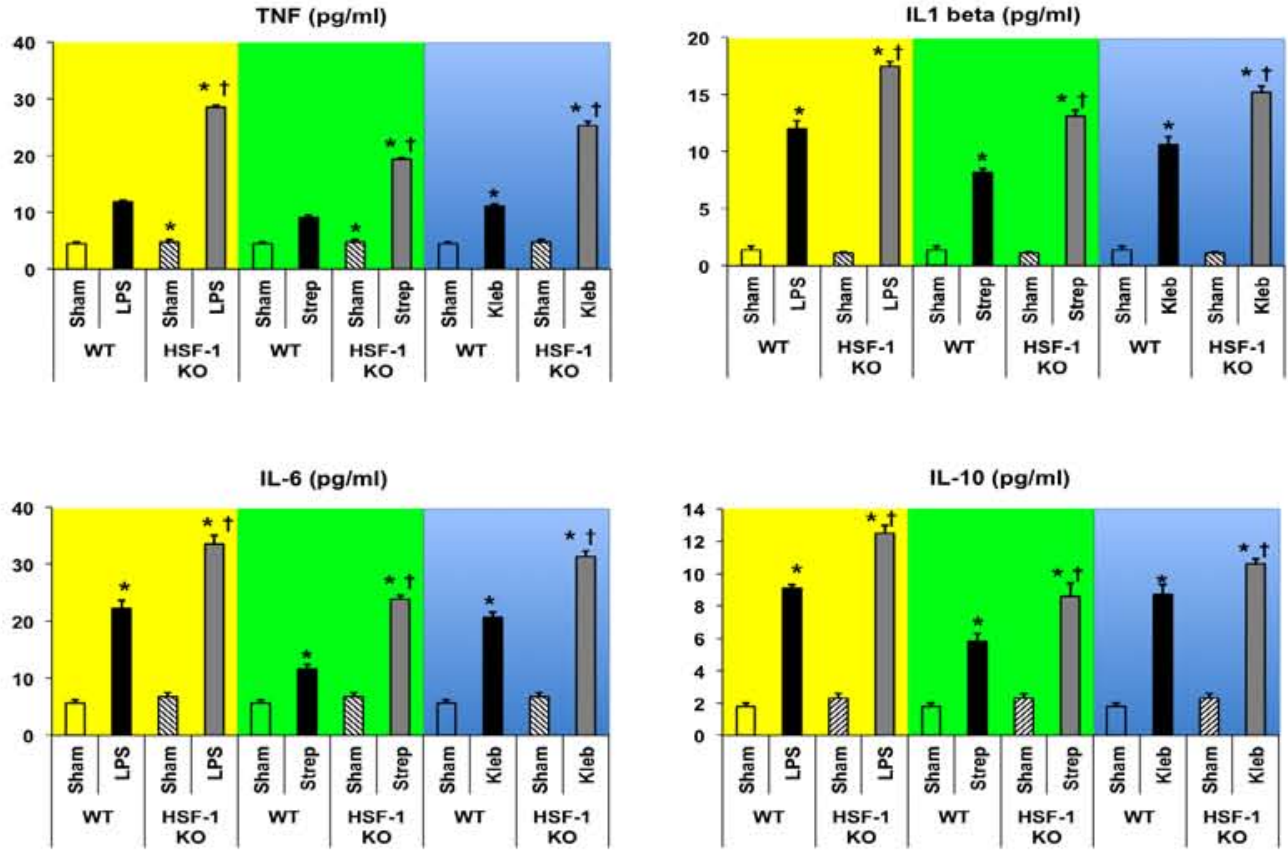

Figure 1: HSF-1 deficiency elevates circulating cytokines in response to septic challenges: Wild type (WT) and HSF-1 knockout mice were subjected to sham or septic challenge by LPS, S. pneumoniae or K. pneumoniae. Levels of TNF, IL-1ß, IL-6 and IL-10 were measured in the blood serum collected 24 hours later. All values are means \pm SE. Statistical significances are labeled with * for a difference between sham and sepsis subjects and $\dagger$ for a difference between HSF-1 KO and wild type, each challenged with LPS, S. pneumoniae or K. pneumoniae (n=6-8 per group).
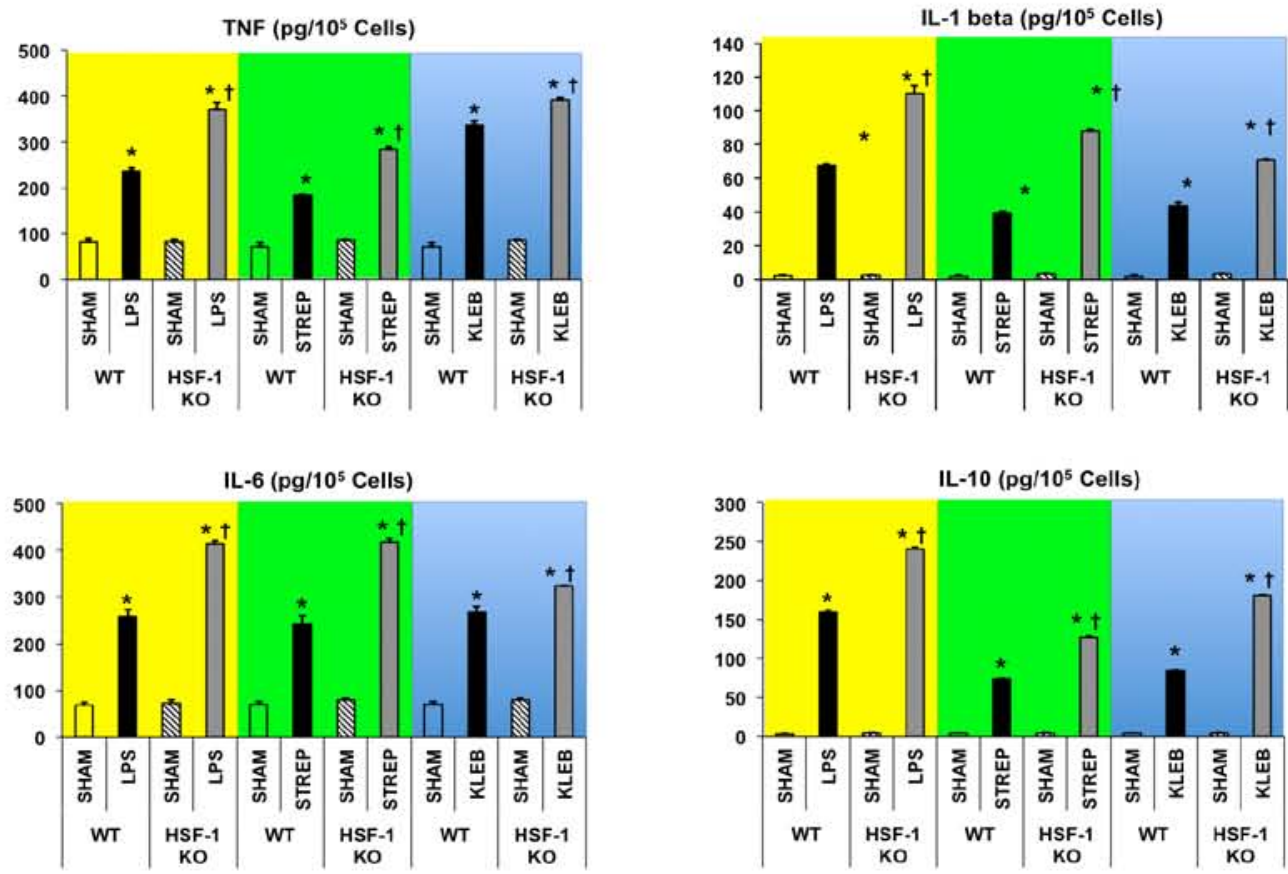

Figure 2: HSF-1 deficiency elevates cytokine production in primary cardiomyocytes from septic challenged mice: Wild type (WT) and HSF-1 knockout mice were subjected to sham or septic challenge by LPS, S. pneumoniae or K. pneumoniae, and cardiomyocytes were isolated from animals sacrificed 24 hours later. Levels of TNF, IL-1 $\beta$, IL-6 and IL-10 were measured in the conditional medium of cardiomyocytes cultured 18 hours after isolation. All values are means \pm SE. Statistical significances are labeled with * for a difference between sham and septic subjects and $\dagger$ for a difference between HSF-1 KO and wild type, each challenged with LPS, S. pneumoniae or K. pneumoniae ( $\mathrm{n}=6-8$ per group). 
A

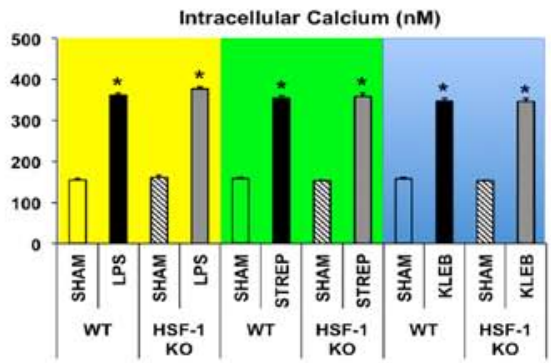

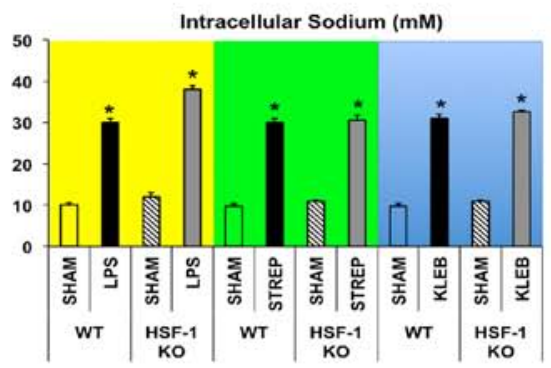

Figure 3: HSF-1 deficiency does not affect intracellular calcium and sodium responses in cardiomyocytes from septic challenged mice: Wild type (WT) and HSF-1 knockout mice were subjected to sham or septic challenge by LPS, S. pneumoniae or K. pneumoniae. Cardiomyocytes were isolated from animals sacrificed 24 hours later, and intracellular levels of calcium and sodium were compared. All values are means \pm SE. Statistical significances are labeled with * for a difference between sham and sepsis subjects ( $\mathrm{n}=6-8$ per group).

wild type and $h s f^{/-}$mice to the Langendorf perfusion to assess cardiac contraction and relaxation [27]. The cardiac stabilization data were collected in hearts perfused at a constant preload, constant heart rate and constant coronary flow rate (Table 1). As summarized in Figure $4,+\mathrm{dP} / \mathrm{dt}$ max, and $-\mathrm{dP} / \mathrm{dt}$ max were similar in all sham animals, regardless of the presence or absence of HSF-1. As expected, giving septic challenge by LPS, S. pneumoniae or K. pneumoniae severely impaired heart contraction and relaxation in wild type mice. Significantly, this sepsis-induced deficiency in left ventricular performance was further exaggerated in the $h s f^{/-}$mice, suggesting that HSF-1 plays a supportive role in maintaining cardiac function during sepsis.

\section{Discussion}

In this study, we observed that septic challenge by LPS, gram-positive bacteria $S$. pneumoniae, or gram-negative bacteria $K$. pneumoniae dramatically elevated systemic and myocardial inflammatory responses, indicated by the rise in production of cytokines TNF, IL-1 $\beta$, IL-6 and IL-10 (Figure 1 and 2). In these animals, all types of septic challenge also produced significant deficits in cardiac contractility, shown by the lower LVP and $\pm \mathrm{dP} / \mathrm{dt}$ max values measured during stabilization of the hearts at a constant preload, constant heart rate, and constant coronary flow rate (Table 1). The sepsis-related LV systolic and diastolic dysfunction was further confirmed by the impaired LVP responses to increase in coronary flow rate (Figure $4 \mathrm{~A}$ ) and increase in perfusate calcium (Figure 4B). Importantly, compared with the wild type mice, $h s f^{\prime-}$ mice exhibited significantly greater increase in cytokine production (Figure 1 and 2) and impairments in LV contraction and relaxation (Figure 4), suggesting that HSF is an important regulatory factor in sepsis-induced inflammation and cardiac dysfunction.

Our data suggest HSF-1 as a regulator of inflammation in the heart during sepsis, since deficiency in HSF-1 expression resulted in further amplified cytokine production in sepsis (Figure 1 and 2); this observation is consistent with previous reports in the literature. Several studies have demonstrated that HSF-1 suppresses certain cytokines through its direct regulation of gene expressions. For example, HSF-1 binds to the 5' promoter of TNF gene to repress TNF transcription [34]. HSF-1 also blocks the transcription of IL-1 $\beta$ gene by interacting with the nuclear factor of IL-6 [35]. Alternatively, HSF-1 may indirectly affect cytokine expression through inactivation of NF- $\kappa \mathrm{B}$, the nuclear factor that regulates the transcription of cytokines such as TNF- $\alpha, I L-1 \beta$, and IL-6. HSF-1 has also been shown to increase degradation of IкB, providing one mechanism by which NF- $\kappa$ B pathway was activated in the HSF-1 deficient models [36]. In septic responses, HSF-1 was suggested to suppress IL-1 $\beta$ expression in LPS-challenged human monocytes [37]. Deficiency in HSF-1 expression in adult mice produced an exaggerated TNF response in the blood and increased mortality rate in response to endotoxin [19]. In this study, we applied multiple septic challenges to $h s f^{1}$ mice and confirmed that HSF-1 functions as an inhibitory modulator in cardiac inflammation during sepsis.

A major heat shock protein (HSP) regulated by HSF-1 is HSP70. HSP70 has been shown to provide cardioprotection via down-regulation of myocardial calcium overload in injury models such as trauma-hemorrhagic shock and ischemia $[38,39]$. The possible mechanism underlying this HSP70 function may involve enhanced expression of calcium transporters such as the ryanodine receptor, sarcoplasmic/endoplasmic reticulum $\mathrm{Ca}^{2+}$-ATPase (SERCA), and $\mathrm{Na}^{+} / \mathrm{Ca}^{2+}$ exchanger (NCX) [38], as well as suppressed activation of p38-MAPKs and/or Raf-1/ ERK pathways [40-42]. In the present study, we observed that intracellular calcium levels measured in both wild type shams and HSF-1 deficient shams were similar (Figure 3). An infectious insult by LPS, gram-positive or gram-negative bacteria elevated calcium loading inside cardiomyocytes. However, this alteration in calcium homeostasis was similar in all groups given a septic challenge, regardless of the presence or absence of HSF-1. These data suggest that intracellular calcium loading was not a major or sustaining cause of the greater myocardial contractile defects measure in HSF-1 deficient mice compared to contractile defects measured in wild type septic mice.

Currently, studies have tightly linked mitochondrial damage with the clinical outcomes of sepsis $[43,44]$. Mitochondrial dysfunction produces overproduction of potentially harmful molecules, such as mitochondrial reactive oxygen species (mtROS) $[45,46]$ and mitochondrial DNA (mtDNA) fragments 
A

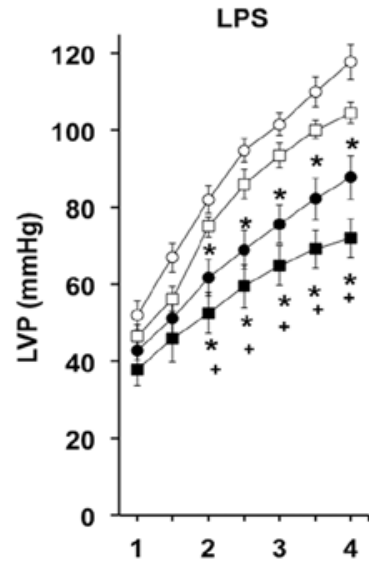

S. PNEUMONIAE

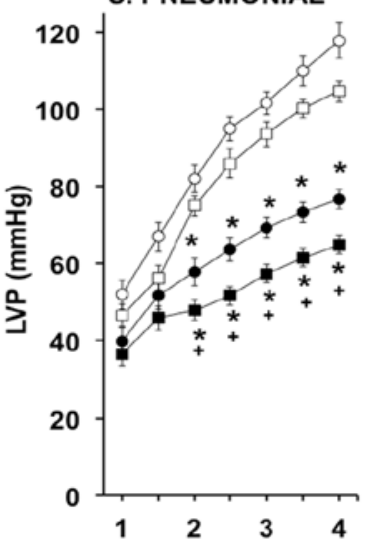

\section{K. PNEUMONIAE}

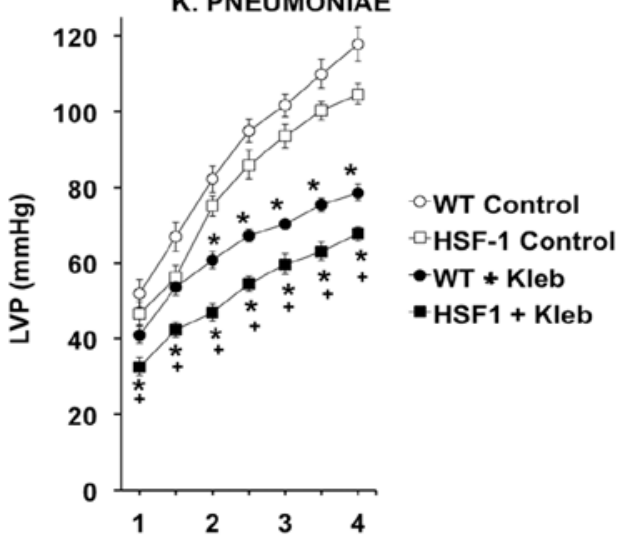

Coronary Flow Rate ( $\mathrm{ml} / \mathrm{min})$

B

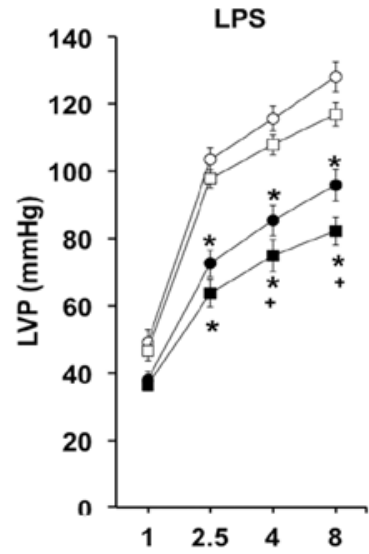

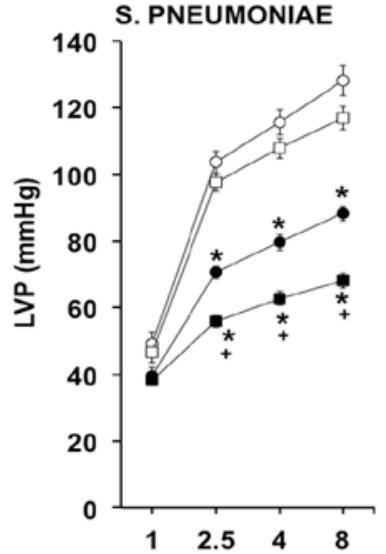

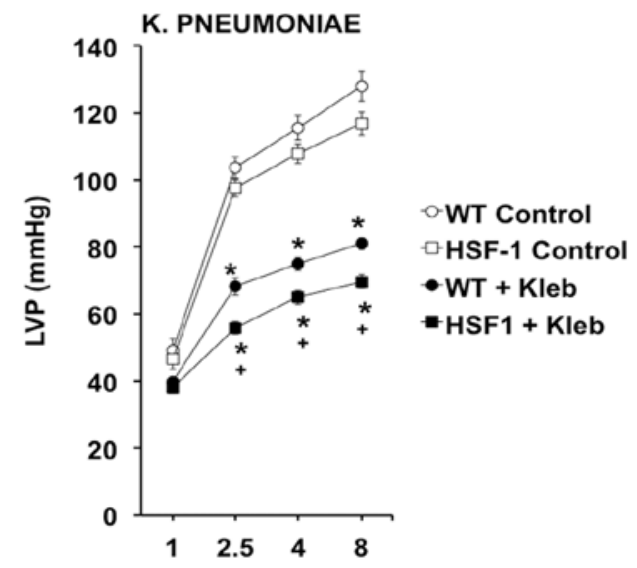

Figure 4: HSF-1 deficiency magnifies sepsis-induced cardiac dysfunction: Wild type (WT) and HSF-1 knockout mice were subjected to sham or septic challenge. 24 hours later, hearts were removed and applied to Langendorff perfusion model for the examination of heart contractility. Data shown are the summary of left ventricular pressure (LVP) responses to incremental increase in coronary flow rate (A) or perfusate calcium (B). All values are means \pm SE. Statistical significances are labeled with $*$ for a difference between sham and sepsis subjects and + for a difference between HSF-1 KO and wild type, each challenged with LPS, S. pneumoniae or K. pneumoniae.

$[47,48]$. These molecules have been observed to escape from impaired mitochondria and function as danger-associated molecular patterns (DAMPs) to simulate inflammatory [49-52], apoptotic [53] and autophagic [54,55] responses. A number of studies showed that HSF-1 and related heat shock proteins play significant roles in the regulation of mitochondria function. It was previously reported that deficiency in HSF-1 expression impaired mitochondrial redox homeostasis and decreased antioxidant defense in the heart [16] and in kidney [56]. Up-regulation of HSP70, a major HSF-1-governed heart shock protein, enhanced mitochondrial superoxide dismutase activity, protected mitochondrial function, and in parallel improved cardiac function after ischemia-reperfusion injury $[57,58]$. We have also previously demonstrated cardiac mitochondrial damage in the sepsis model [21]. Therefore, we suspect that a deficiency of HSF1 expression further impairs myocardial mitochondrial structure and function, which at least in part leads to the intensified cardiac dysfunction in septic $h s f^{\%}$ mice. However, this hypothesis needs to be further tested in our future studies.

In summary, data obtained in a mouse sepsis model suggest that HSF-1 and related heat shock protein members are essential cardiac protective factors. TNF, IL-1 secretion by cardiac myocytes after LPS, S. pneumoniae, or $K$. pneumoniae challenge was exaggerated in the HSF-1 deficient mice compared to responses measured in wild type counter parts. This enhanced systemic and myocardial inflammation 
Deficiency in Heat Shock Factor 1 (HSF-1) Expression Exacerbates Sepsis-induced Inflammation and Cardiac Dysfunction

\begin{tabular}{|c|c|c|c|c|c|c|c|c|c|c|c|c|}
\hline & \multicolumn{2}{|c|}{ Wild Type } & \multicolumn{2}{|c|}{ HSF-1 K0 } & \multicolumn{2}{|c|}{ Wild Type } & \multicolumn{2}{|c|}{ HSF-1 K0 } & \multicolumn{2}{|c|}{ Wild Type } & \multicolumn{2}{|c|}{ HSF-1 K0 } \\
\hline & Sham & LPS & Sham & LPS & Vehicle & Strep & Vehicle & Strep & Vehicle & Kleb & Vehicle & Kleb \\
\hline LVP (mmHg) & $97.5 \pm 2.3$ & $62.8 \pm 2.0^{*}$ & $98 \pm 3.1$ & $61.1 \pm 4.6^{*}$ & $97.5 \pm 2.3$ & $66.3 \pm 2.9^{*}$ & $98 \pm 3.1$ & $52.6 \pm 1.8 * \dagger$ & $97.5 \pm 2.3$ & $67.3 \pm 2.4$ & $98 \pm 3.1$ & $53.0 \pm 1.0 * \dagger$ \\
\hline $\begin{array}{c}+\mathrm{dP} / \mathrm{dt} \\
(\mathrm{mmHg} / \mathrm{sec})\end{array}$ & $2308 \pm 60$ & $1606 \pm 83^{*}$ & $2348 \pm 211$ & $1466 \pm 167^{*}$ & $2308 \pm 60$ & $1729 \pm 58 *$ & $2348 \pm 211$ & $1337 \pm 52 * \dagger$ & $2308 \pm 60$ & $1638 \pm 90$ & $2348 \pm 211$ & $1364 \pm 62 * \dagger$ \\
\hline $\begin{array}{c}-\mathrm{dP} / \mathrm{dt} \\
(\mathrm{mmHg} / \mathrm{sec})\end{array}$ & $1940 \pm 189$ & $1229 \pm 65^{*}$ & $2018 \pm 69$ & $1228 \pm 156^{*}$ & $1940 \pm 189$ & $1367 \pm 104^{*}$ & $2018 \pm 69$ & $1092 \pm 66^{*}+$ & $1940 \pm 189$ & $1416 \pm 95$ & $2018 \pm 69$ & $1104 \pm 80^{*}+$ \\
\hline $\mathrm{dP40}$ & $1965 \pm 59$ & $1406 \pm 88^{*}$ & $1955 \pm 47$ & $1200 \pm 148^{*}$ & $1965 \pm 59$ & $1517 \pm 58^{*}$ & $1955 \pm 47$ & $1150 \pm 44 * \dagger$ & $1965 \pm 59$ & $1383 \pm 95$ & $1955 \pm 47$ & $1157 \pm 49 * \dagger$ \\
\hline TPP (msec) & $76 \pm 2.6$ & $74.4 \pm 1.6$ & $79.7 \pm 2.4$ & $74 \pm 3.7$ & $76 \pm 2.6$ & $71.8 \pm 2.9$ & $79.7 \pm 2.4$ & $73 \pm 3.9$ & $76 \pm 2.6$ & $77.2 \pm 3.1$ & $79.7 \pm 2.4$ & $73.9 \pm 1.6$ \\
\hline RT90 (msec) & $79.9 \pm 1.3$ & $77.1 \pm 1.9$ & $78.6 \pm 2.0$ & $78.2 \pm 2.8$ & $79.9 \pm 1.3$ & $82.3 \pm 3.1$ & $78.6 \pm 2$ & $74.9 \pm 2.3$ & $79.9 \pm 1.3$ & $79.3 \pm 2.3$ & $78.6 \pm 2$ & $77.0 \pm 2.6$ \\
\hline $\begin{array}{l}\mathrm{TM}+\mathrm{dP} / \mathrm{dt} \\
\text { (msec) }\end{array}$ & $50.1 \pm 1.2$ & $49.8 \pm 0.3$ & $53.5 \pm 1.7$ & $48 \pm 1.3$ & $50.1 \pm 1.2$ & $47.1 \pm 1.4$ & $53.5 \pm 1.7$ & $47.3 \pm 1.9$ & $50.1 \pm 1.2$ & $50.7 \pm 1.5$ & $53.5 \pm 1.7$ & $49.7 \pm 0.3$ \\
\hline $\begin{array}{c}\mathrm{TM}-\mathrm{dP} / \mathrm{dt} \\
\text { (msec) }\end{array}$ & $51.3 \pm 0.9$ & $49.8 \pm 0.5$ & $52.6 \pm 1.4$ & $51.1 \pm 1.3$ & $51.3 \pm 0.9$ & $53.4 \pm 1.1$ & $52.6 \pm 1.4$ & $51.1 \pm 1.8$ & $51.3 \pm 0.9$ & $51.7 \pm 1.7$ & $52.6 \pm 1.4$ & $50.9 \pm 1.7$ \\
\hline CPP (mmHg) & $87.9 \pm 8.4$ & $71.3 \pm 8.1$ & $82 \pm 3.9$ & $75.3 \pm 9.0$ & $87.9 \pm 8.4$ & $92.8 \pm 5.3$ & $82 \pm 3.9$ & $73.3 \pm 8.5$ & $87.9 \pm 8.4$ & $67 \pm 9.4$ & $82 \pm 3.9$ & $76.9 \pm 9.5$ \\
\hline $\begin{array}{c}\text { CVR } \\
(\mathrm{mmHg} / \mathrm{sec})\end{array}$ & $58.6 \pm 5.6$ & $47.4 \pm 5.4$ & $54.7 \pm 2.3$ & $50.2 \pm 6.0$ & $58.6 \pm 5.6$ & $61.9 \pm 3.5$ & $54.7 \pm 2.3$ & $48.9 \pm 5.6$ & $58.6 \pm 5.6$ & $44.7 \pm 6.2$ & $54.7 \pm 2.3$ & $51.2 \pm 6.3$ \\
\hline $\begin{array}{c}\text { HR } \\
\text { (beats/min) }\end{array}$ & $333 \pm 12$ & $330 \pm 10$ & $325 \pm 17$ & $318 \pm 13$ & $333 \pm 12$ & $360 \pm 10$ & $325 \pm 17$ & $355 \pm 13$ & $333 \pm 12$ & $323 \pm 9$ & $325 \pm 17$ & $337 \pm 10$ \\
\hline
\end{tabular}

Table 1: HSF-1 deficiency magnifies sepsis-induced cardiac dysfunction.

LVP, left ventricular pressure; +dP/dt, the rate of LVP rise; -dP/dt, the rate of LVP fall; $\mathrm{dP} 40, \mathrm{dP} / \mathrm{dt}$ value at a developed pressure of $40 \mathrm{mmHg} / \mathrm{sec}$; $\mathrm{TPP}$, time to peak pressure; RT90, time to $90 \%$ relaxation; TM $+\mathrm{dP} / \mathrm{dt}$, time to $\max +\mathrm{dP} / \mathrm{dt}$; TM $-\mathrm{dP} / \mathrm{dt}$, time to $\max -\mathrm{dP} / \mathrm{dt}$;

$\mathrm{CPP}$, coronary perfusion pressure; CVR, coronary vascular resistance; HR, heart rate. All values are means \pm SE. Statistical significances are labeled with

* for a difference between sham and sepsis subjects and $\dagger$ for a difference between HSF-1 KO and wild type, each challenged with LPS, Streptococcus pneumoniae (Strep) or Klebsiella pneumoniae (Kleb). ( $p<0.05, \mathrm{n}=4-6$ per group).

was associated with significantly greater deficiency in cardiac contraction and relaxation. However, we also acknowledge that a few limitations in the current report should be addressed in future investigations. First, we chose to study TNF, IL-1, IL-1 $\beta$, IL-6 and IL-10 as inflammation markers. Whether HSF-1 has any regulatory effect on the generation of other cytokines and DAMP molecules such as high-mobility group box 1 (HMGB1) [59] and hyaluronan fragments [60] will help us to further reveal the mechanism with regards to HSF-1-mediated cardiac protection during sepsis. Second, we did not examine whether lacking HSF-1 affects sepsis survival in our animal models, due to the tremendous effort to obtain relatively large quantity of animals that are needed to establish statistical significance. However, our future research effort will be extended to examine the role of HSF-1 in other organ functions in the mouse sepsis model. We believe these investigations will assist to validate whether HSF-1 and related heat shock proteins are potential therapeutic targets to encounter multiple organ dysfunction syndrome (MODS) in sepsis.

\section{Acknowledgement}

This work was supported by NIH Grant R01 GM057054-08 and 5 P50 GM 216181-41.

\section{References}

1. Angus DC, Pereira CA, Silva E (2006) Epidemiology of severe sepsis around the world. Endocr Metab Immune Disord Drug Targets 6: 207212.

2. Bone RC, Balk RA, Cerra FB, Dellinger RP, Fein AM, et al. (1992) Definitions for sepsis and organ failure and guidelines for the use of innovative therapies in sepsis. The ACCP/SCCM Consensus Conference
Committee. American College of Chest Physicians/Society of Critical Care Medicine. Chest 101: 1644-1655.

3. Levy MM, Dellinger RP, Townsend SR, Linde-Zwirble WT, Marshall JC, et al. The Surviving Sepsis Campaign: results of an international guideline-based performance improvement program targeting severe sepsis. Crit Care Med 38: 367-374.

4. O'Brien JM, Jr., Ali NA, Aberegg SK, Abraham E (2007) Sepsis. Am J Med 120: 1012-1022.

5. Venkataraman R, Subramanian S, Kellum JA (2003) Clinical review: extracorporeal blood purification in severe sepsis. Crit Care 7: 139145 .

6. Lovat R, Preiser JC (2003) Antioxidant therapy in intensive care. Curr Opin Crit Care 9: 266-270.

7. Court 0, Kumar A, Parrillo JE (2002) Clinical review: Myocardial depression in sepsis and septic shock. Crit Care 6: 500-508.

8. Zanotti-Cavazzoni SL, Hollenberg SM (2009) Cardiac dysfunction in severe sepsis and septic shock. Curr Opin Crit Care 15: 392-397.

9. Rudiger A, Singer M (2007) Mechanisms of sepsis-induced cardiac dysfunction. Crit Care Med 35: 1599-1608.

10.Tanonaka K, Yoshida H, Toga W, Furuhama K, Takeo S (2001) Myocardial heat shock proteins during the development of heart failure. Biochem Biophys Res Commun 283: 520-525.

11. Rafiee P, Shi Y, Pritchard KA, Jr., Ogawa H, Eis AL, et al. (2003) Cellular redistribution of inducible Hsp70 protein in the human and rabbit heart in response to the stress of chronic hypoxia: role of protein kinases. J Biol Chem 278: 43636-43644.

12. Horton JW, Maass DL, White DJ, Sanders B, Murphy J (2004) Effects of burn serum on myocardial inflammation and function. Shock 22: 438-445.

13. Gabai VL, Meriin AB, Yaglom JA, Wei JY, Mosser DD, et al. (2000) 
Suppression of stress kinase JNK is involved in HSP72-mediated protection of myogenic cells from transient energy deprivation. HSP72 alleviates the stewss-induced inhibition of JNK dephosphorylation. J Biol Chem 275: 38088-38094.

14. Huot J, Lambert H, Lavoie JN, Guimond A, Houle F, et al. (1995) Characterization of 45-kDa/54-kDa HSP27 kinase, a stress-sensitive kinase which may activate the phosphorylation-dependent protective function of mammalian 27-kDa heat-shock protein HSP27. Eur J Biochem 227: 416-427.

15. Wei H, Campbell W, Vander Heide RS (2006) Heat shock-induced cardioprotection activates cytoskeletal-based cell survival pathways. Am J Physiol Heart Circ Physiol 291: H638-647.

16. Yan LJ, Christians ES, Liu L, Xiao X, Sohal RS, et al. (2002) Mouse heat shock transcription factor 1 deficiency alters cardiac redox homeostasis and increases mitochondrial oxidative damage. EMBO J 21: 5164-5172.

17. Voellmy R (2004) On mechanisms that control heat shock transcription factor activity in metazoan cells. Cell Stress Chaperones 9: 122-133.

18. Morimoto RI, Santoro MG (1998) Stress-inducible responses and heat shock proteins: new pharmacologic targets for cytoprotection. Nat Biotechnol 16: 833-838.

19. Xiao X, Zuo X, Davis AA, McMillan DR, Curry BB, et al. (1999) HSF1 is required for extra-embryonic development, postnatal growth and protection during inflammatory responses in mice. EMBO J 18: 59435952.

20. McMillan DR, Xiao X, Shao L, Graves K, Benjamin IJ (1998) Targeted disruption of heat shock transcription factor 1 abolishes thermotolerance and protection against heat-inducible apoptosis. J Biol Chem 273: 7523-7528.

21.Zang Q, Maass DL, Tsai SJ, Horton JW (2007) Cardiac mitochondrial damage and inflammation responses in sepsis. Surg Infect (Larchmt) 8: 41-54.

22. Zang QS, Martinez B, Yao X, Maass DL, Ma L, et al. (2012) Sepsis-Induced Cardiac Mitochondrial Dysfunction Involves Altered MitochondrialLocalization of Tyrosine Kinase Src and Tyrosine Phosphatase SHP2. PLoS One 7: e43424.

23.Zang QS, Sadek H, Maass DL, Martinez B, Ma L, et al. (2012) Specific inhibition of mitochondrial oxidative stress suppresses inflammation and improves cardiac function in a rat pneumonia-related sepsis model. Am J Physiol Heart Circ Physiol 302: H1847-1859.

24. Horton JW, Maass DL, White J, Sanders B (2001) Hypertonic saline-dextran suppresses burn-related cytokine secretion by cardiomyocytes. Am J Physiol Heart Circ Physiol 280: H1591-1601.

25.Zhang H, Wang HY, Bassel-Duby R, Maass DL, Johnston WE, et al. (2007) Role of interleukin-6 in cardiac inflammation and dysfunction after burn complicated by sepsis. Am J Physiol Heart Circ Physiol 292: H2408-2416.

26.Zang QS, Maass DL, Wigginton JG, Barber RC, Martinez B, et al. (2010) Burn serum causes a CD14-dependent mitochondrial damage in primary cardiomyocytes. Am J Physiol Heart Circ Physiol 298: H19511958.

27.Zang Q, Maass DL, White J, Horton JW (2007) Cardiac mitochondrial damage and loss of ROS defense after burn injury: the beneficial effects of antioxidant therapy. J Appl Physiol 102: 103-112.

28. White DJ, Carlson D, Ordway GA, Horton JW (2004) Protective role of heat stress in burn trauma. Crit Care Med 32: 1338-1345.
29. White J, Maass DL, Giroir B, Horton JW (2001) Development of an acute burn model in adult mice for studies of cardiac function and cardiomyocyte cellular function. Shock 16: 122-129.

30. Horton JW, Tan J, White DJ, Maass DL, Thomas JA (2004) Selective decontamination of the digestive tract attenuated the myocardial inflammation and dysfunction that occur with burn injury. Am J Physiol Heart Circ Physiol 287: H2241-2251.

31. Sikes PJ, Zhao P, Maass DL, White J, Horton JW (2005) Sodium/ hydrogen exchange activity in sepsis and in sepsis complicated by previous injury: 31P and 23Na NMR study. Crit Care Med 33: 605-615.

32. Maass DL, White J, Sanders B, Horton JW (2005) Role of cytosolic vs. mitochondrial $\mathrm{Ca} 2+$ accumulation in burn injury-related myocardial inflammation and function. Am J Physiol Heart Circ Physiol 288: H744-751.

33. Maass DL, White DJ, Sanders B, Horton JW (2005) Cardiac myocyte accumulation of calcium in burn injury: cause or effect of myocardial contractile dysfunction. J Burn Care Rehabil 26: 252-259.

34. Singh IS, He JR, Calderwood S, Hasday JD (2002) A high affinity HSF1 binding site in the 5 -untranslated region of the murine tumor necrosis factor-alpha gene is a transcriptional repressor. J Biol Chem 277: 4981-4988.

35. Xie Y, Chen C, Stevenson MA, Auron PE, Calderwood SK (2002) Heat shock factor 1 represses transcription of the IL-1beta gene through physical interaction with the nuclear factor of interleukin 6. J Biol Chem 277: 11802-11810.

36. Wirth D, Bureau F, Melotte D, Christians E, Gustin P (2004) Evidence for a role of heat shock factor 1 in inhibition of NF-kappaB pathway during heat shock response-mediated lung protection. Am J Physiol Lung Cell Mol Physiol 287: L953-961.

37. Cahill CM, Waterman WR, Xie Y, Auron PE, Calderwood SK (1996) Transcriptional repression of the prointerleukin 1beta gene by heat shock factor 1. J Biol Chem 271: 24874-24879.

38. Liu J, Kam KW, Borchert GH, Kravtsov GM, Ballard HJ, et al. (2006) Further study on the role of HSP70 on $\mathrm{Ca} 2+$ homeostasis in rat ventricular myocytes subjected to simulated ischemia. Am J Physiol Cell Physiol 290: C583-591.

39. Yu HP, Shimizu T, Choudhry MA, Hsieh YC, Suzuki T, et al. (2006) Mechanism of cardioprotection following trauma-hemorrhagic shock by a selective estrogen receptor-beta agonist: up-regulation of cardiac heat shock factor-1 and heat shock proteins. J Mol Cell Cardiol 40: 185194.

40. Kim YK, Suarez J, Hu Y, McDonough PM, Boer C, et al. (2006) Deletion of the inducible $70-\mathrm{kDa}$ heat shock protein genes in mice impairs cardiac contractile function and calcium handling associated with hypertrophy. Circulation 113: 2589-2597.

41. Liu J, Kam KW, Zhou JJ, Yan WY, Chen M, et al. (2004) Effects of heat shock protein 70 activation by metabolic inhibition preconditioning or kappa-opioid receptor stimulation on $\mathrm{Ca} 2+$ homeostasis in rat ventricular myocytes subjected to ischemic insults. J Pharmacol Exp Ther 310: 606-613.

42. Zhou JJ, Pei JM, Wang GY, Wu S, Wang WP, et al. (2001) Inducible HSP70 mediates delayed cardioprotection via $\mathrm{U}-50488 \mathrm{H}$ pretreatment in rat ventricular myocytes. Am J Physiol Heart Circ Physiol 281: H40-47.

43. Brealey D, Brand M, Hargreaves I, Heales S, Land J, et al. (2002) Association between mitochondrial dysfunction and severity and outcome of septic shock. Lancet 360: 219-223. 
44. Cairns CB, Moore FA, Haenel JB, Gallea BL, Ortner JP, et al. (1997) Evidence for early supply independent mitochondrial dysfunction in patients developing multiple organ failure after trauma. J Trauma 42 532-536.

45. Beal MF (2002) Oxidatively modified proteins in aging and disease. Free Radic Biol Med 32: 797-803.

46. Croteau DL, Bohr VA (1997) Repair of oxidative damage to nuclear and mitochondrial DNA in mammalian cells. J Biol Chem 272: 25409 25412.

47. Rothfuss O, Gasser T, Patenge N (2010) Analysis of differential DNA damage in the mitochondrial genome employing a semi-long run realtime PCR approach. Nucleic Acids Res 38: e24.

48. Yakes FM, Van Houten B (1997) Mitochondrial DNA damage is more extensive and persists longer than nuclear DNA damage in human cells following oxidative stress. Proc Natl Acad Sci U S A 94: 514-519.

49.Zhang Q, Raoof M, Chen Y, Sumi Y, Sursal T, et al. (2010) Circulating mitochondrial DAMPs cause inflammatory responses to injury. Nature 464: 104-107.

50. Tschopp J (2011) Mitochondria: Sovereign of inflammation? Eur J Immunol 41: 1196-1202.

51. Krysko DV, Agostinis P, Krysko O, Garg AD, Bachert C, et al. (2011) Emerging role of damage-associated molecular patterns derived from mitochondria in inflammation. Trends Immunol 32: 157-164.

52. Koenitzer JR, Freeman BA (2010) Redox signaling in inflammation: interactions of endogenous electrophiles and mitochondria in cardiovascular disease. Ann N Y Acad Sci 1203: 45-52.

53. Handy DE, Loscalzo J (2012) Redox regulation of mitochondrial function. Antioxid Redox Signal 16: 1323-1367.

54. Wrighton KH (2011) Autophagy: shaping the fate of mitochondria. Nat Rev Mol Cell Biol 12: 344-345.

55.Scherz-Shouval R, Elazar Z (2007) ROS, mitochondria and the regulation of autophagy. Trends Cell Biol 17: 422-427.

56. Yan LJ, Rajasekaran NS, Sathyanarayanan S, Benjamin IJ (2005) Mouse HSF1 disruption perturbs redox state and increases mitochondrial oxidative stress in kidney. Antioxid Redox Signal 7: 465-471.

57. Suzuki K, Murtuza B, Sammut IA, Latif N, Jayakumar J, et al. (2002) Heat shock protein 72 enhances manganese superoxide dismutase activity during myocardial ischemia-reperfusion injury, associated with mitochondrial protection and apoptosis reduction. Circulation 106: I270-276.

58. Jayakumar J, Suzuki K, Sammut IA, Smolenski RT, Khan M, et al. (2001) Heat shock protein 70 gene transfection protects mitochondrial and ventricular function against ischemia-reperfusion injury. Circulation 104: I303-307.

59. Scaffidi P, Misteli T, Bianchi ME (2002) Release of chromatin protein HMGB1 by necrotic cells triggers inflammation. Nature 418: 191-195.

60. Scheibner KA, Lutz MA, Boodoo S, Fenton MJ, Powell JD, et al. (2006) Hyaluronan fragments act as an endogenous danger signal by engaging TLR2. J Immunol 177: 1272-1281. 